Document downloaded from:

http://hdl.handle.net/10251/66350

This paper must be cited as:

Cordero Barbero, A.; Lotfi, T.; Bakhtiari, P.; Torregrosa Sánchez, JR. (2015). An efficient two-parametric family with memory for nonlinear equations. Numerical Algorithms.

68(2):323-335. doi:10.1007/s11075-014-9846-8.

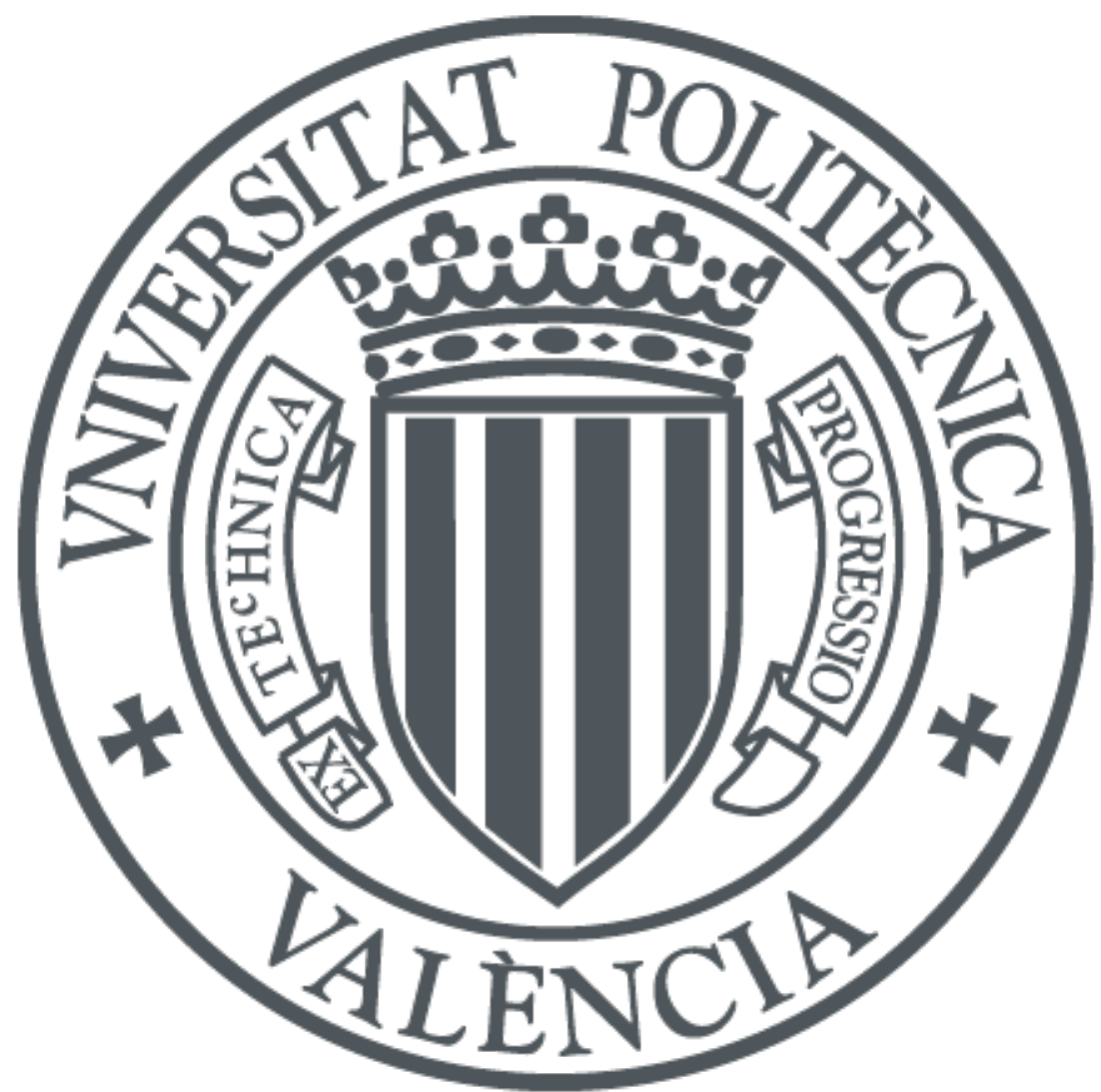

The final publication is available at

http://dx.doi.org/10.1007/s11075-014-9846-8

Copyright Springer Verlag (Germany)

Additional Information 


\title{
An efficient two-parametric family with memory for nonlinear equations *
}

\author{
Alicia Cordero ${ }^{a, \dagger} \quad$ Taher Lotfi $^{b, \ddagger} \quad$ Parisa Bakhtiari $^{c, \S} \quad$ Juan R. Torregrosa $^{a}{ }^{\uparrow}$ \\ ${ }^{a}$ Instituto de Matemática Multidisciplinar, Universitat Politècnica de València, \\ Camino de Vera, s/n, 46022 Valencia, Spain \\ ${ }^{b}$ Department of Applied Mathematics, Hamedan Branch, Islamic Azad University, \\ Hamedan, Iran \\ ${ }^{c}$ Department of Mathematics, Imam Khomeini International University, Ghazvin, 34149-16818, Iran.
}

\begin{abstract}
A new two-parametric family of derivative-free iterative methods for solving nonlinear equations is presented. First, a new biparametric family without memory of optimal order four is proposed. The improvement of the convergence rate of this family is obtained by using two self-accelerating parameters. These varying parameters are calculated in each iterative step employing only information from the current and the previous iteration. The corresponding $R$-order is 7 and the efficiency index $7^{1 / 3}=1.913$. Numerical examples and comparison with some existing derivative-free optimal eighth-order schemes are included to confirm the theoretical results. In addition, the dynamical behavior of the designed method is analyzed and shows the stability of the scheme.
\end{abstract}

Keywords: Multipoint iterative method; nonlinear equation; optimal order; method with memory, Kung-Traub's conjecture.

\section{Introduction}

Solving nonlinear equations is a classical problem which has interesting applications in various branches of science and engineering. In this study, we describe new iterative methods to find a simple root $\alpha$ of a nonlinear equation $f(x)=0$, where $f: I \subseteq \mathbb{R} \rightarrow \mathbb{R}$ is a scalar function on an open interval $I$.

The most efficient existing root-solvers are based on multipoint iterations. This class of methods overcomes theoretical limits of one-point schemes related to the convergence order and the efficiency index. However, the Kung-Traub's conjecture limits the order of convergence of a multipoint method without memory. Kung and Traub conjectured in [1] that the order of convergence of any of these schemes can not exceed the bound $2^{d-1}$, where $d$ is the number of functional evaluations per step. When this bound is achieved the method is called optimal. We recall that, commonly, the efficiency of an iterative method is measured by the efficiency index defined as $E=p^{1 / d}$, where $p$ is the order of convergence.

Many high-order multipoint methods without memory have been already derived in the literature by using different procedures: divided differences, weight functions, frozen derivative, interpolation polynomials, ..., see for instance $[2,3,4,5,6,7,8,9,10,12,13]$ and the references therein.

In this paper, from the derivative-free method presented by Zheng et al. in [13] we design a biparametric family of two-point optimal fourth-order methods without memory. The convergence rate of this family is significantly increased by applying an accelerating procedure based on varying two parameters calculated by Newton's interpolating polynomials in each iteration. This accelerating technique relies on information from the current and the previous iterative step, defining in this way two-point methods with memory. The increase of convergence speed is achieved without additional functional evaluations, which is an important advantage of these methods compared to other known ones.

\footnotetext{
*This research was supported by Ministerio de Ciencia y Tecnología MTM2011-28636-C02-02

†acordero@mat.upv.es

${ }_{\ddagger}^{\ddagger}$ lotfi@iauh.ac.ir, lotfitaher@yahoo.com

§akhtiari@iauh.ac.ir, bakhtiaripr@yahoo.ac.ir

`Corresponding author: jrtorre@mat.upv.es
} 
In the following, we use the symbols $\rightarrow, O$ and $\sim$ according to the following conventions [11]: If $\lim _{n \rightarrow \infty} g\left(x_{n}\right)=$ $C$, we write $g\left(x_{n}\right) \rightarrow C$ or $g \rightarrow C$. If $\lim _{x \rightarrow \alpha} g(x)=C$, we write $g(x) \rightarrow C$ or $g \rightarrow C$. If $\frac{f}{g} \rightarrow C$, where $C$ is a nonzero constant, we write $f=O(g)$ or $f \sim C g$.

The rest of the paper is organized as follows: in Section 2 we describe the biparametric family of Steffensentype two-point iterative schemes and we prove the fourth-order of convergence, with independence of the values of the parameters. Section 3 is devoted to design the method with memory and to prove its R-order and its efficiency index. The numerical study presented in Section 4 confirm the theoretical results and the excellent convergence properties of the presented method in comparison with some optimal iterative method, without memory, of order 8. These good convergence properties are confirmed with the dynamical study carried out in this section.

\section{Derivative-free two-point family}

Zheng et al. in [13] presented a Steffensen-type iterative method with fourth-order of convergence. Its iterative expression is

$$
\left\{\begin{array}{l}
y_{k}=x_{k}-\frac{f\left(x_{k}\right)}{f\left[x_{k}, w_{k}\right]}, \quad w_{k}=x_{k}+\gamma f\left(x_{k}\right), \quad \gamma \neq 0 \\
x_{k+1}=y_{k}-\frac{f\left(y_{k}\right)}{f\left[x_{k}, y_{k}\right]+\left(y_{k}-x_{k}\right) f\left[x_{k}, w_{k}, y_{k}\right]}
\end{array}\right.
$$

where $f[x, y]=\frac{f(x)-f(y)}{x-y}$ and $f[x, w, y]=\frac{f[x, w]-f[w, y]}{x-y}$ are the divided differences of order 1 and 2, respectively.

Let us consider the following modification of method (1) with an additional parameter $\lambda$ :

$$
\left\{\begin{array}{l}
y_{k}=x_{k}-\frac{f\left(x_{k}\right)}{f\left[x_{k}, w_{k}\right]+\lambda f\left(w_{k}\right)}, \quad w_{k}=x_{k}+\gamma f\left(x_{k}\right), \quad \lambda \text { and } \gamma \neq 0, \\
x_{k+1}=y_{k}-\frac{f\left(y_{k}\right)}{f\left[x_{k}, y_{k}\right]+\left(y_{k}-x_{k}\right) f\left[x_{k}, w_{k}, y_{k}\right]} .
\end{array}\right.
$$

For $k \geq 0$, we introduce the following notation for the different errors:

$$
e_{k}=x_{k}-\alpha, \quad e_{k, w}=w_{k}-\alpha, \quad e_{k, y}=y_{k}-\alpha
$$

and

$$
c_{i}=\frac{1}{i !} \frac{f^{(i)}(\alpha)}{f^{\prime}(\alpha)}, \quad i=2,3, \ldots
$$

In order to obtain the order of convergence and the error equation of family (2), and also to avoid rather cumbersome expressions that appear in a standard convergence analysis of iterative methods, we are going to employ symbolic computation in the computational software package Mathematica. The following abbreviation are used

$$
\mathrm{f} 1 \mathrm{a}=\mathrm{f}^{\prime}(\alpha), \mathrm{e}=\mathrm{x}-\alpha, \quad \mathrm{e}_{\mathrm{w}}=\mathrm{w}-\alpha, \quad \mathrm{e}_{\mathrm{y}}=\mathrm{y}-\alpha, \quad \mathrm{e}_{\hat{\mathrm{x}}}=\hat{\mathrm{x}}-\alpha, \quad \mathrm{c}_{\mathrm{i}}=\frac{\mathrm{f}^{(\mathrm{i})}(\alpha)}{\mathrm{i} ! \mathrm{f}^{\prime}(\alpha)} \quad \mathrm{i}=2,3, \ldots
$$

Program written in Mathematica

$$
\begin{aligned}
& \operatorname{In}[1]: \quad \mathrm{f}\left[\mathrm{e}_{-}\right]:=\mathrm{f} 1 \mathrm{a}\left(\mathrm{e}+\sum_{\mathrm{i}=2}^{4} \mathrm{c}_{\mathrm{i}} * \mathrm{e}^{\mathrm{i}}\right) \\
& \operatorname{In}[2]: \quad e_{\mathrm{w}}:=\mathrm{e}+\gamma \operatorname{Series}[\mathrm{f}[\mathrm{e}], \mathrm{e}, 0,4] / / \text { FullSimplify } \\
& \text { out }[2]: \quad(1+\gamma f 1 \mathrm{a}) \mathrm{e}+\mathrm{O}[\mathrm{e}]^{2} \\
& \operatorname{In}[3]: \quad \mathrm{f}\left[\mathrm{x}_{-}, \mathrm{y}_{-}\right]:=\frac{\mathrm{f}[\mathrm{x}]-\mathrm{f}[\mathrm{y}]}{\mathrm{x}-\mathrm{y}} \text {; } \\
& \operatorname{In}[4]: \quad \mathrm{f}\left[\mathrm{x}_{-}, \mathrm{y}_{-}, \mathrm{z}_{-}\right]:=\frac{\mathrm{f}[\mathrm{x}, \mathrm{y}]-\mathrm{f}[\mathrm{y}, \mathrm{z}]}{\mathrm{x}-\mathrm{z}} \text {; } \\
& \operatorname{In}[5]: \quad e_{y}=e-\frac{f[e]}{f\left[e, e_{w}\right]+\lambda f\left[e_{w}\right]} / / \text { FullSimplify } \\
& \text { Out [5]: } \quad(1+\gamma f 1 \mathrm{a})\left(\lambda+\mathrm{c}_{2}\right) \mathrm{e}^{2}+\mathrm{O}[\mathrm{e}]^{3} \\
& \operatorname{In}[6]: \quad e_{\hat{x}}=e_{y}-\frac{f\left[e_{y}\right]}{f\left[e, e_{y}\right]+\left(e_{y}-e\right) f\left[e, e_{w}, e_{y}\right]} / / \text { FullSimplify } \\
& \text { out [6]: } \quad(1+\gamma f 1 a)^{2}\left(\lambda+c_{2}\right)\left(c_{2}\left(\lambda+c_{2}\right)-c_{3}\right) e^{4}+0[e]^{5} \text {. }
\end{aligned}
$$

Remark 2.1. In the above outputs, we have just reported the first terms of the corresponding error equations as they are required later (see the proof of Theorem 3.2).

Therefore, we can establish the following result. 
Theorem 2.2. Let $\alpha \in I$ be a simple root of a sufficiently differentiable function $f: I \subseteq \mathbb{R} \rightarrow \mathbb{R}$ in an open interval I. If $x_{0}$ is close enough to $\alpha$, then the order of convergence of the class of two-step methods (2) is at least four and its error equation is given by

$$
e_{k+1}=\left(1+\gamma f^{\prime}(\alpha)\right)^{2}\left(\lambda+c_{2}\right)\left(c_{2}\left(\lambda+c_{2}\right)-c_{3}\right) e_{k}^{4}+O\left(e_{k}^{5}\right) .
$$

\section{The development of a new method with memory}

In this section, we are going to design from family (2), a new method with memory by using two self-accelerating parameters. We observe from (3) that the order of convergence of the family (2) is four when $\gamma \neq-1 / f^{\prime}(\alpha)$ and $\lambda \neq-c_{2}$. With the choice $\gamma=-1 / f^{\prime}(\alpha)$ and $\lambda=-c_{2}=-f^{\prime \prime}(\alpha) /\left(2 f^{\prime}(\alpha)\right)$, it can be proved that the order of the family (2) can reach 7, being $e_{k+1}=-c_{2}^{2} c_{3}^{2} e_{k}^{7}+O\left(e_{k}^{8}\right)$ its error equation. But the values of $f^{\prime}(\alpha)$ and $f^{\prime \prime}(\alpha)$ are unknown, so the idea in constructing methods with memory consists of the calculation of the parameters $\gamma=\gamma_{k}$ and $\lambda=\lambda_{k}$ as the iteration proceeds by the formulas $\gamma_{k}=-1 / \tilde{f}^{\prime}(\alpha)$ and $\lambda_{k}=-\tilde{c_{2}}=-\tilde{f}^{\prime \prime}(\alpha) /\left(2 \tilde{f}^{\prime}(\alpha)\right)$ for $k=1,2, \ldots$, where $\tilde{f}^{\prime}$ and $\tilde{c_{2}}$ are aproximations to $f^{\prime}(\alpha)$ and $c_{2}$, respectively.

We consider the following accelerator for approximating the parameters $\gamma_{k}$ and $\lambda_{k}$

$$
\gamma_{k}=\frac{-1}{\tilde{f}^{\prime}(\alpha)}=\frac{-1}{N_{3}^{\prime}\left(x_{k}\right)}, \quad \lambda_{k}=-\frac{N_{4}^{\prime \prime}\left(w_{k}\right)}{2 N_{4}^{\prime}\left(w_{k}\right)}
$$

where

$$
N_{3}(t)=N_{3}\left(t ; x_{k}, y_{k-1}, x_{k-1}, w_{k-1}\right) \text { and } N_{4}(t)=N_{4}\left(t ; w_{k}, x_{k}, y_{k-1}, w_{k-1}, x_{k-1}\right)
$$

are Newton's interpolating polynomials of third and fourth degree, set through four and five best available approximations (nodes) $x_{k}, y_{k-1}, x_{k-1}, w_{k-1}$ and $w_{k}, x_{k}, y_{k-1}, x_{k-1}, w_{k-1}$, respectively. It should be noted that if one uses lower degree Newton's interpolation, lower accelerators are obtained. It is assumed that initial estimates $\gamma_{0}$ and $\lambda_{0}$ should be chosen before starting the iterative process.

Replacing the fixed parameter $\gamma$ and $\lambda$ in the iterative formula (2) by the varying $\gamma_{k}$ and $\lambda_{k}$ calculated by (4), the following derivative-free two-points scheme with memory is achieved:

$$
\left\{\begin{array}{l}
x_{0}, \gamma_{0}, \lambda_{0} \text { are given, then } w_{0}=x_{0}+\gamma_{0} f\left(x_{0}\right), \\
\gamma_{k}=-\frac{1}{N_{3}^{\prime}\left(x_{k}\right)}, \quad w_{k}=x_{k}+\gamma_{k} f\left(x_{k}\right), \quad \lambda_{k}=-\frac{N_{4}^{\prime \prime}\left(w_{k}\right)}{2 N_{4}^{\prime}\left(w_{k}\right)}, \quad k=1,2, \ldots \\
y_{k}=x_{k}-\frac{f\left(x_{k}\right)}{f\left[w_{k}, x_{k}\right]+\lambda_{k} f\left(w_{k}\right)}, \\
x_{k+1}=y_{k}-\frac{f\left(y_{k}\right)}{f\left[x_{k}, y_{k}\right]+\left(y_{k}-x_{k}\right) f\left[x_{k}, w_{k}, y_{k}\right]} .
\end{array}\right.
$$

In the next subsection we are going to prove the convergence of the scheme (5) by using the concept and the notation of $R$-order, introduced in [14].

\subsection{Convergence analysis}

To obtain the order of convergence of the two-point scheme with memory (5), we need the following technical result whose proof is obtained by using the error of Newton's interpolation, in a similar way as Lemma 1 of [4].

Lemma 3.1. If $\gamma_{k}=-1 / N_{3}^{\prime}\left(x_{k}\right)$ and $\lambda_{k}=-N_{4}^{\prime \prime}\left(w_{k}\right) /\left(2 N_{4}^{\prime}\left(w_{k}\right)\right), k=1,2, \ldots$, then the estimates

$$
1+\gamma_{k} f^{\prime}(\alpha) \sim c_{4} e_{k-1, y} e_{k-1, w} e_{k-1} \quad \text { and } \quad c_{2}+\lambda_{k} \sim \frac{3 c_{5}}{2 c_{2}} e_{k-1, y} e_{k-1, w} e_{k-1}
$$

hold.

Now we state the following convergence result for the scheme (5):

Theorem 3.2. If an initial estimation $x_{0}$ is close enough to a simple root $\alpha$ of $f(x)=0$, being $f$ a real sufficiently differentiable function, then the R-order of convergence of the two-point method with memory (5) is at least 7. 
Proof. Let $\left\{x_{k}\right\}$ be a sequence of approximations generated by an iterative method (IM). If this sequence converges to a root $\alpha$ of $f(x)=0$ with the $R$-order $O_{R}((I M), \alpha) \geq R$, we will write

$$
e_{k+1} \sim D_{k, R} e_{k}^{R}, \quad e_{k}=x_{k}-\alpha,
$$

where $D_{k, R}$ tends to the asymptotic error constant $D_{R}$ of (IM), when $k \rightarrow \infty$. Hence

$$
e_{k+1} \sim D_{k, R} e_{k}^{R}=D_{k, R}\left(D_{k-1, R} e_{k-1}^{R}\right)^{R}=D_{k, R} D_{k-1, R} e_{k-1}^{R^{2}} .
$$

We assume that the $R$-order of the iterative sequences $\left\{w_{k}\right\}$ and $\left\{y_{k}\right\}$ are at least $p$ and $q$, respectively, that is,

$$
e_{k, w} \sim D_{k, p} e_{k}^{p}=D_{k, p}\left(D_{k-1, R} e_{k-1}^{R}\right)^{p}=D_{k, p} D_{k-1, R}^{p} e_{k-1}^{R p}
$$

and

$$
e_{k, y} \sim D_{k, q} e_{k}^{q}=D_{k, q}\left(D_{k-1, R} e_{k-1}^{R}\right)^{q}=D_{k, q} D_{k-1, R}^{q} e_{k-1}^{R q} .
$$

Considering these R-orders and Lemma 3.1, we obtain

$$
\begin{aligned}
1+\gamma_{k} f^{\prime}(\alpha) & \sim c_{4} e_{k-1, w} e_{k-1, y} e_{k-1}=c_{4} D_{k-1, p} e_{k-1}^{p} D_{k-1, q} e_{k-1}^{q} e_{k-1}=c_{4} D_{k-1, p} D_{k-1, q} e_{k-1}^{p+q+1}, \\
c_{2}+\lambda_{k} & \sim \frac{3 c_{5}}{2 c_{2}} e_{k-1, w} e_{k-1, y} e_{k-1}=\frac{3 c_{5}}{2 c_{2}} D_{k-1, p} e_{k-1}^{p} D_{k-1, q} e_{k-1}^{q} e_{k-1}=\frac{3 c_{5}}{2 c_{2}} D_{k-1, p} D_{k-1, q} e_{k-1}^{p+q+1},
\end{aligned}
$$

or

$$
\begin{aligned}
1+\gamma_{k} f^{\prime}(\alpha) & \sim c_{4} D_{k-1, p} D_{k-1, q} e_{k-1}^{p+q+1}, \\
c_{2}+\lambda_{k} & \sim \frac{3 c_{5}}{2 c_{2}} D_{k-1, p} D_{k-1, q} e_{k-1}^{p+q+1} .
\end{aligned}
$$

By Theorem 2.2, see remark 2.1, it can be inferred that

$$
\begin{aligned}
& e_{k, w} \sim\left(1+\gamma_{k} f^{\prime}(\alpha)\right) e_{k}, \\
& e_{k, y} \sim c_{2}\left(1+\gamma_{k} f^{\prime}(\alpha)\right)\left(c_{2}+\lambda_{k}\right) e_{k}^{2}, \\
& e_{k+1} \sim\left(1+\gamma_{k} f^{\prime}(\alpha)\right)^{2}\left(c_{2}+\lambda_{k}\right) e_{k}^{4} .
\end{aligned}
$$

Consequently, combining (11)-(15), yield

$$
\begin{gathered}
e_{k, w} \sim\left(1+\gamma_{k} f^{\prime}(\alpha)\right)\left(c_{2}+\lambda_{k}\right) e_{k} \sim c_{4} D_{k-1, p} D_{k-1, q} D_{k-1, R} e_{k-1}^{(1+p+q)+R}, \\
e_{k, y} \sim c_{2}\left(1+\gamma_{k} f^{\prime}(\alpha)\right)\left(c_{2}+\lambda_{k}\right) e_{k}^{2} \sim c_{2} c_{4} D_{k-1, p} D_{k-1, q} D_{k-1, R}^{2} e_{k-1}^{2(1+p+q)+2 R},
\end{gathered}
$$

and

$$
e_{k+1} \sim A_{4}\left(1+\gamma_{k} f^{\prime}(\alpha)\right)^{2}\left(c_{2}+\lambda_{k}\right) e_{k}^{4} \sim A_{4} c_{4} D_{k-1, p}^{2} D_{k-1, q}^{2} D_{k-1, R}^{4} e_{k-1}^{3(1+p+q)+4 R} .
$$

By comparing the powers of error exponents of $e_{k-1}$ in pairs of relations (9)-(16), (10)-(17) and (8)-(18), we obtain the nonlinear system of three equations with unknowns $p, q$ and $R$

$$
\left\{\begin{array}{l}
R p-R-(p+q+1)=0, \\
R q-2 R-2(p+q+1)=0, \\
R^{2}-4 R-3(p+q+1)=0 .
\end{array}\right.
$$

The unique positive solution of this system is given by $p=2, q=4$ and $R=7$, which define the order of convergence of the derivative-free scheme with memory (5).

We have proved that the order of convergence of the iterative scheme (5) is, at least, 7. So, its efficiency index is $7^{\frac{1}{3}}=1.913$ which is greater than that of the optimal methods of order eight, $8^{\frac{1}{4}}=1.682$. In the next section, we are going to compare scheme (5) with some derivative-free optimal eighth-order methods. 


\section{Numerical results and dynamical behavior}

In order to show the efficiency of the described method, we will compare its features with the ones obtained by optimal derivative-free eighth-order methods without memory, both numerical and dynamically.

Derivative-free Zheng et al. method, see [13], denoted by ZLH8, has the iterative expression

$$
\begin{aligned}
y_{k} & =x_{k}-\frac{f\left(x_{k}\right)}{f\left[x_{k}, w_{k}\right]}, \quad w_{k}=x_{k}-f\left(x_{k}\right), \\
u_{k} & =y_{k}-\frac{f\left(y_{k}\right)}{f\left[x_{k}, y_{k}\right]+\left(y_{k}-x_{k}\right) f\left[y_{k}, x_{k}, w_{k}\right]}, \\
x_{k+1} & =u_{k}-\frac{f\left(u_{k}\right)}{f\left[u_{k}, y_{k}\right]+f\left[u_{k}, y_{k}, x_{k}\right]\left(u_{k}-y_{k}\right)+f\left[u_{k}, y_{k}, x_{k}, w_{k}\right]\left(u_{k}-y_{k}\right)\left(u_{k}-x_{k}\right)},
\end{aligned}
$$

where $f[u, y, x, w]$ is the divided difference of order three.

Steffensen-type procedure by Thukral et al., see [10], is denoted by T8 and it is expressed as

$$
\begin{aligned}
y_{k} & =x_{k}-\frac{f\left(x_{k}\right)}{f\left[x_{k}, w_{k}\right]}, \quad w_{k}=x_{k}-f\left(x_{k}\right), \\
u_{k} & =y_{k}-\frac{f\left(y_{k}\right) f\left[z_{k}, x_{k}\right]}{f\left[x_{k}, y_{k}\right] f\left[y_{k}, w_{k}\right]}, \\
x_{k+1} & =u_{k}-\frac{f\left(u_{k}\right)}{f\left[u_{k}, y_{k}\right]+f\left[x_{k}, y_{k}\right]+f\left[x_{k}, u_{k}\right]}\left(1-\frac{f\left(u_{k}\right)}{f\left(w_{k}\right)}\right)^{-1}\left(1+\frac{2 f\left(y_{k}\right)^{3}}{f\left(w_{k}\right)^{2} f\left(x_{k}\right)}\right)^{-1} .
\end{aligned}
$$

Finally, the derivative-free method, by Soleymani et al. [8], denoted by S8, has the iterative expression

$$
\begin{aligned}
y_{k}= & x_{k}-\frac{f\left(x_{k}\right)}{f\left[x_{k}, w_{k}\right]}, \quad w_{k}=x_{k}-f\left(x_{k}\right), \\
u_{k}= & y_{k}-\frac{f\left(y_{k}\right) f\left(w_{k}\right)}{f\left[x_{k}, y_{k}\right]\left(f\left(w_{k}\right)-f\left(y_{k}\right)\right)}, \\
x_{k+1}= & u_{k}-\frac{f\left(u_{k}\right) f\left(w_{k}\right)}{f\left[x_{k}, y_{k}\right]\left(f\left(w_{k}\right)-f\left(y_{k}\right)\right)}\left(1+\frac{f\left(u_{k}\right)}{f\left(y_{k}\right)}\right) \\
& \left(1+\frac{f\left(u_{k}\right)}{f\left(w_{k}\right)}\right)\left(1+\frac{f\left(u_{k}\right)}{f\left(x_{k}\right)}\right)\left(1+\left(1+f\left[x_{k}, w_{k}\right]\right)\left(\frac{f\left(y_{k}\right)}{f\left(w_{k}\right)}\right)^{2}\right) .
\end{aligned}
$$

All of them have order of convergence eight and are optimal schemes, in the sense of Kung-Traub's conjecture, without memory.

In the following we will compare the numerical results obtained by the proposed seventh-order scheme with memory with optimal eight-order ones (without memory). Our goal is to show the high efficiency and stability of the proposed method, even compared with optimal ones with higher order of convergence.

The errors $\left|x_{k}-\alpha\right|$ of approximations to the sought roots, produced by the different methods at the first three iterations, are given in Tables 1 and 2, where $A e-h$ stands for $A \times 10^{-h}$. These tables also include, for each test function, the initial estimation values and the last value of the computational order of convergence $\rho$ (see [15]) computed by the expression (if it is stable)

$$
\rho=\frac{\log \left(\left|f\left(x_{k}\right) / f\left(x_{k-1}\right)\right|\right)}{\log \left(\left|f\left(x_{k-1}\right) / f\left(x_{k-2}\right)\right|\right)} .
$$

The software Mathematica 8, with 2000 arbitrary precision arithmetic has been used in our computations. The test functions used are:

(1) $f_{1}(x)=\frac{1}{3} x^{4}-x^{2}-\frac{1}{3} x+1, \quad \alpha \approx 1.54682 \ldots$,

(2) $f_{2}(x)=(x-2)\left(x^{6}+x^{3}+1\right) e^{-x^{2}}, \quad \alpha=2$,

(3) $f_{3}(x)=e^{x^{3}-x}-\cos \left(x^{2}-1\right)+x^{3}+1, \quad \alpha=-1$,

(4) $f_{4}(x)=\tan ^{2} x, \quad \alpha=0$ double root, 
Table 1: Numerical tests with $f_{1}$ and $f_{2}$

\begin{tabular}{|l|c|c|c|c|}
\hline & M7 & ZLH8 & T8 & S8 \\
\hline \hline$f_{1}, x_{0}=3$ & & & & \\
$\left|x_{1}-\alpha\right|$ & 0.3470 & 0.5037 & 0.3880 & 0.5365 \\
$\left|x_{2}-\alpha\right|$ & $0.2120 \mathrm{e}-3$ & $0.7172 \mathrm{e}-1$ & $0.2078 \mathrm{e}-1$ & $0.9544 \mathrm{e}-1$ \\
$\left|x_{3}-\alpha\right|$ & $0.9429 \mathrm{e}-25$ & $0.7718 \mathrm{e}-4$ & $0.1151 \mathrm{e}-8$ & $0.1269 \mathrm{e}-3$ \\
$\rho$ & 6.0572 & 2.5492 & 4.5783 & 2.7148 \\
\hline$f_{1}, x_{0}=2$ & & & & \\
$\left|x_{1}-\alpha\right|$ & $0.4224 \mathrm{e}-1$ & $0.5635 \mathrm{e}-1$ & $0.3370 \mathrm{e}-1$ & $0.6434 \mathrm{e}-1$ \\
$\left|x_{2}-\alpha\right|$ & $0.2255 \mathrm{e}-8$ & $0.2408 \mathrm{e}-4$ & $0.2361 \mathrm{e}-7$ & $0.1199 \mathrm{e}-4$ \\
$\left|x_{3}-\alpha\right|$ & $0.3010 \mathrm{e}-59$ & $0.4889 \mathrm{e}-24$ & $0.1119 \mathrm{e}-55$ & $0.1008 \mathrm{e}-33$ \\
$\rho$ & 6.9527 & 5.7439 & 7.8063 & 7.6579 \\
\hline$f_{2}, x_{0}=1.5$ & & & & \\
$\left|x_{1}-\alpha\right|$ & $0.2813 \mathrm{e}-1$ & 1.739 & 1.703 & 1.343 \\
$\left|x_{2}-\alpha\right|$ & $0.1550 \mathrm{e}-10$ & 2.156 & 2.988 & 1.918 \\
$\left|x_{3}-\alpha\right|$ & $0.9025 \mathrm{e}-76$ & 2.506 & 2.988 & 2.353 \\
$\rho$ & 7.0573 & - & - & - \\
\hline$f_{2}, x_{0}=1.8$ & & & & \\
$\left|x_{1}-\alpha\right|$ & $0.1361 \mathrm{e}-2$ & $0.2033 \mathrm{e}-5$ & $0.1981 \mathrm{e}-5$ & $0.3482 \mathrm{e}-4$ \\
$\left|x_{2}-\alpha\right|$ & $0.1409 \mathrm{e}-20$ & $0.3923 \mathrm{e}-32$ & $0.1405 \mathrm{e}-42$ & $0.6602 \mathrm{e}-32$ \\
$\left|x_{3}-\alpha\right|$ & $0.5493 \mathrm{e}-146$ & $0.2028 \mathrm{e}-192$ & $0.8968 \mathrm{e}-340$ & $0.1103 \mathrm{e}-253$ \\
$\rho$ & 6.9727 & 6.0000 & 8.0000 & 8.0000 \\
\hline \multicolumn{5}{|l}{} \\
\hline
\end{tabular}

(5) $f_{5}(x)=(x-1)^{3}-1, \quad \alpha=2$.

The results alongside the test functions are given in Tables 1 and 2, calculated with the initial values $\gamma_{0}=\lambda_{0}=$ -0.01 in the proposed scheme.

It is known that the good selection of initial estimations plays a decisive role in iterative methods, but specially in derivative-free ones. One of the advantages showed in these numerical tests is that the dependence on the proximity of the initial approximation is not as important in M7 as in optimal eighth-order schemes without memory. This makes M7 converge when the other methods fail. However, there is no advantage in other standard problems, as in multiple roots, but neither are disadvantages. In general, it can be stated from Tables 1 and 2 that M7 shows a good stability and convergence properties.

From the numerical point of view, the dynamical behavior of the rational function associated with an iterative method gives us important information about its stability and reliability.

We are going to recall now some dynamical concepts (see [16]) that we use in this work. Given a rational function $R: \hat{\mathbb{C}} \rightarrow \hat{\mathbb{C}}$, where $\hat{\mathbb{C}}$ is the Riemann sphere, the orbit of a point $z_{0} \in \hat{\mathbb{C}}$ is defined as:

$$
\left\{z_{0}, R\left(z_{0}\right), R^{2}\left(z_{0}\right), \ldots, R^{n}\left(z_{0}\right), \ldots\right\} .
$$

We analyze the phase plane of the map $R$ by classifying the starting points from the asymptotic behavior of their orbits. A $z_{0} \in \hat{\mathbb{C}}$ is called a fixed point if $R\left(z_{0}\right)=z_{0}$. Moreover, a fixed point $z_{0}$ is called attractor if $\left|R^{\prime}\left(z_{0}\right)\right|<1$, superattractor if $\left|R^{\prime}\left(z_{0}\right)\right|=0$, repulsor if $\left|R^{\prime}\left(z_{0}\right)\right|>1$ and parabolic if $\left|R^{\prime}\left(z_{0}\right)\right|=1$. Then, the basin of attraction of an attractor $\alpha$ is defined as:

$$
\mathcal{A}(\alpha)=\left\{z_{0} \in \hat{\mathbb{C}}: R^{n}\left(z_{0}\right) \rightarrow \alpha, n \rightarrow \infty\right\} .
$$

Also, it is well-known that the basin of attraction of any fixed point belongs to the so called Fatou set and the boundaries of these basins of attraction are the Julia set.

In the following, we study the stability properties of the proposed method for some of the functions involved in the numerical test. In fact, we show the dynamical planes associated to the rational function obtained when the method is applied on functions $f_{2}(z)$ and $f_{5}(z)$. These planes are obtained in the following way: in the rectangle $[-1.5,2.5] \times[-2,2]$ of the complex plane, a mesh of $400 \times 400$ initial estimations is defined. If the sequence generated by the iterative method reaches a root of the function (superattracting fixed point) with an error estimation lower than $10^{-3}$ and a maximum of 40 iterations, we decide that the initial point is in the basin of attraction of these root and we paint it in a color previously selected for this root. The roots of each function are marked with a white star. In the same basin of attraction, the number of iterations needed to achieve the solution 
Table 2: Numerical tests with $f_{3}$ to $f_{5}$

\begin{tabular}{|l|c|c|c|c|}
\hline & M7 & ZLH8 & T8 & S8 \\
\hline \hline$f_{3}, x_{0}=-1.65$ & & & & \\
$\left|x_{1}-\alpha\right|$ & $0.8448 \mathrm{e}-3$ & $0.7177 \mathrm{e}-2$ & $0.1463 \mathrm{e}-3$ & $0.1495 \mathrm{e}-3$ \\
$\left|x_{2}-\alpha\right|$ & $0.3751 \mathrm{e}-18$ & $0.1261 \mathrm{e}-12$ & $0.2468 \mathrm{e}-28$ & $0.3540 \mathrm{e}-27$ \\
$\left|x_{3}-\alpha\right|$ & $0.7054 \mathrm{e}-130$ & $0.5922 \mathrm{e}-77$ & $0.1610 \mathrm{e}-226$ & $0.3466 \mathrm{e}-216$ \\
$\rho$ & 7.2774 & 5.9804 & 8.0001 & 8.0001 \\
\hline$f_{3}, x_{0}=-2$ & & & & \\
$\left|x_{1}-\alpha\right|$ & 0.5495 & 0.4250 & 0.5500 & $0.1736 \mathrm{e}-1$ \\
$\left|x_{2}-\alpha\right|$ & $0.2163 \mathrm{e}-1$ & 81.16 & 0.5263 & $0.5124 \mathrm{e}-11$ \\
$\left|x_{3}-\alpha\right|$ & $0.3119 \mathrm{e}-11$ & - & $0.3880 \mathrm{e}-2$ & $0.6688 \mathrm{e}-87$ \\
$\rho$ & 8.3593 & - & - & 7.9603 \\
\hline$f_{4}, x_{0}=-0.5$ & & & & \\
$\left|x_{1}-\alpha\right|$ & 0.1358 & $0.6815 \mathrm{e}-1$ & $0.2990 \mathrm{e}-1$ & $0.5027 \mathrm{e}-1$ \\
$\left|x_{2}-\alpha\right|$ & $0.1389 \mathrm{e}-1$ & $0.1252 \mathrm{e}-1$ & $0.3875 \mathrm{e}-2$ & $0.8197 \mathrm{e}-2$ \\
$\left|x_{3}-\alpha\right|$ & $0.1392 \mathrm{e}-2$ & $0.2339 \mathrm{e}-2$ & $0.5098 \mathrm{e}-3$ & $0.1364 \mathrm{e}-2$ \\
$\rho$ & 1.0063 & 0.9897 & 0.9926 & 0.9882 \\
\hline$f_{4}, x_{0}=0.8$ & & & & \\
$\left|x_{1}-\alpha\right|$ & 0.2415 & 0.2616 & 0.1955 & 0.3238 \\
$\left|x_{2}-\alpha\right|$ & $0.6840 \mathrm{e}-2$ & $0.5722 \mathrm{e}-1$ & $0.2881 \mathrm{e}-1$ & $0.6762 \mathrm{e}-1$ \\
$\left|x_{3}-\alpha\right|$ & $0.7002 \mathrm{e}-3$ & $0.1096 \mathrm{e}-1$ & $0.3859 \mathrm{e}-2$ & $0.1171 \mathrm{e}-1$ \\
$\rho$ & 0.6360 & 1.0724 & 1.0429 & 1.0969 \\
\hline$f_{5}, x_{0}=1.5$ & & & & \\
$\left|x_{1}-\alpha\right|$ & 0.1345 & 14.0400 & 3.7670 & $0.1260 \mathrm{e}+8$ \\
$\left|x_{2}-\alpha\right|$ & $0.5325 \mathrm{e}-7$ & 6.7950 & 1.0680 & $0.6309 \mathrm{e}+7$ \\
$\left|x_{3}-\alpha\right|$ & $0.1349 \mathrm{e}-51$ & 3.5580 & $0.9752 \mathrm{e}-1$ & $0.3159 \mathrm{e}+7$ \\
$\rho$ & 6.9041 & 0.9884 & 1.2211 & - \\
\hline$f_{5}, x_{0}=3.5$ & & & & \\
$\left|x_{1}-\alpha\right|$ & 0.1991 & 0.2718 & 0.2172 & 0.3522 \\
$\left|x_{2}-\alpha\right|$ & $0.6470 \mathrm{e}-6$ & $0.2175 \mathrm{e}-2$ & $0.7302 \mathrm{e}-4$ & $0.5889 \mathrm{e}-2$ \\
$\left|x_{3}-\alpha\right|$ & $0.5272 \mathrm{e}-44$ & $0.6610 \mathrm{e}-14$ & $0.8318 \mathrm{e}-30$ & $0.2549 \mathrm{e}-14$ \\
$\rho$ & 6.8360 & 5.2153 & 7.2787 & 6.4464 \\
\hline
\end{tabular}

is showed in darker or brighter colors. Black color denotes lack of convergence to any of the roots (with the maximum of iterations established) or convergence to the infinity. These dynamical planes have been generated by using the software showed in [17], implemented in Matlab R2011a.

The same iterative methods that appeared in the numerical section have been used for dynamical purposes: the basins of attraction of these schemes on functions $f_{2}(z)$ and $f_{5}(z)$ are drawn in Figure 1 and 2, respectively, showing their stability and the complexity of their associated Julia and Fatou sets.

Let us observe that the basin of the roots are, in general, bigger for method M7. This fact justifies the better convergence of the method when the initial estimation is not very close to the solution, as happened in the numerical section. The behavior of this scheme is much more stable than the others, as can be seen in Figures 1a and 2a. We think that the reason of this improvement in the stability is the introduction of the memory factors (M7 method) on lower order schemes, versus high-order methods without memory.

\section{Conclusions}

From a new optimal biparametric fourth-order family, we have designed a derivative-free seventh-order method with memory which has an efficiency index higher than those of optimal eight-order procedure without memory. Numerical and dynamical features have been checked. The results obtained showed that method M7 converges to the root even when the initial estimation is far from the solution. This is also showed in the dynamical planes, as the basins of attraction of M7 are wider than those of the optimal eighth-order schemes used to compare. 


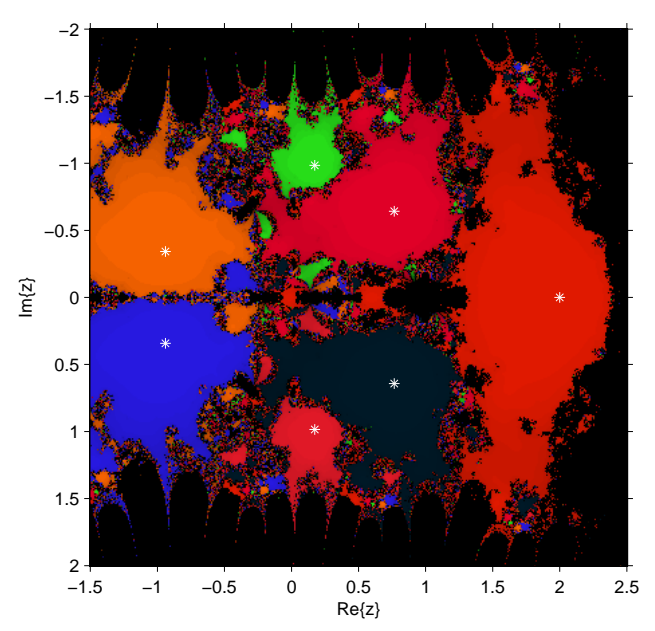

(a) M7

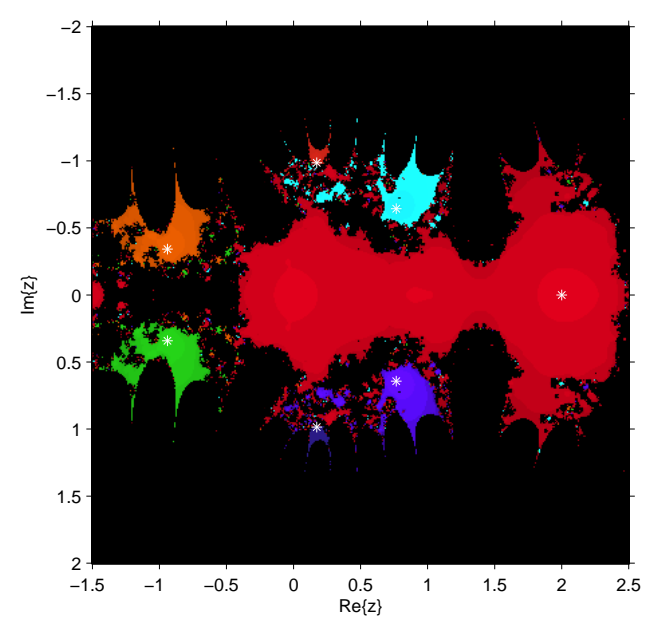

(c) $\mathrm{T} 8$

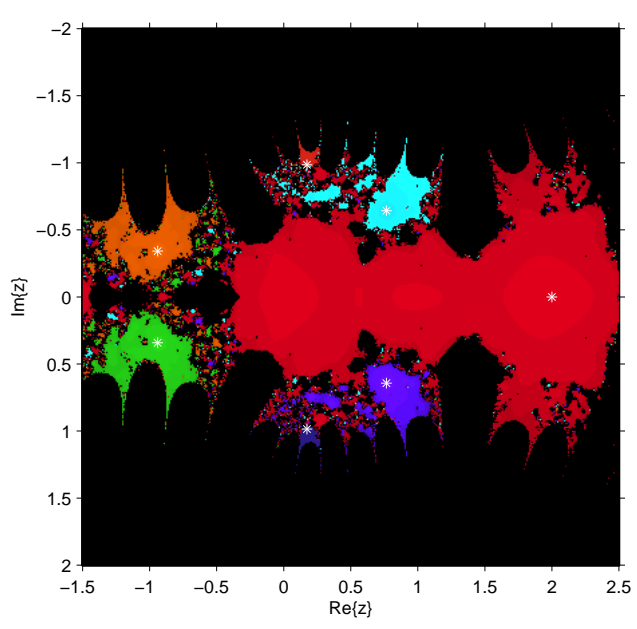

(b) ZLH8

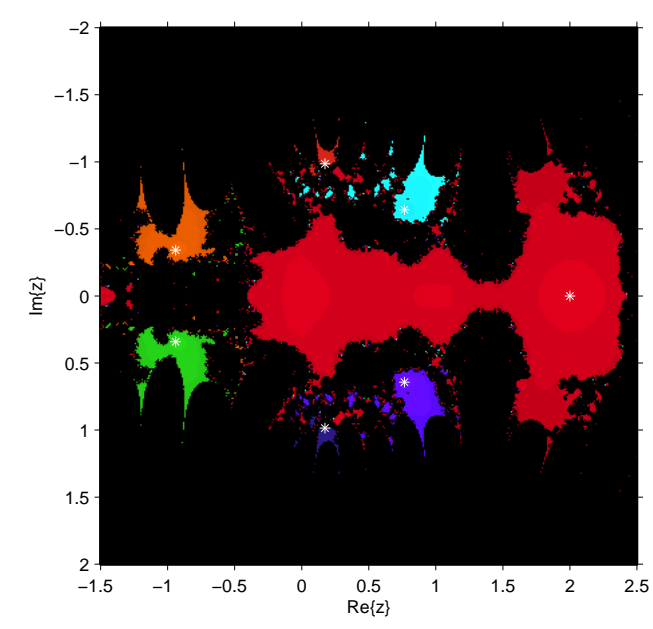

(d) S8

Figure 1: Dynamical planes of different schemes on $f_{2}(z)=(z-2)\left(z^{6}+z^{3}+1\right) e^{-z^{2}}$

\section{Acknowlegment}

We would like to express our gratitude to the anonymous referees for their insightful valuable comments and suggestions.

The second author wishes to thank the Islamic Azad University, Hamedan Branch, where the paper was written as a part of the research plan, for financial support.

\section{References}

[1] H. T. Kung, J. F. Traub, Optimal order of one-point and multi-point iteration, J. Assoc. Comput. Math. 21, 643-651 (1974)

[2] A. Cordero, J.L. Hueso, E. Martínez, J. R. Torregrosa, A new technique to obtain derivative-free optimal iterative methods for solving nonlinear equation, Journal of Computational and Applied Mathematics 252, 95-102 (2013)

[3] A. Cordero, J. R. Torregrosa, M. P. Vassileva, Pseudocomposition: A technique to design predictor-corrector methods for systems of nonlinear equations, Applied Mathematics and Computation 218, 11496-11508 (2012) 


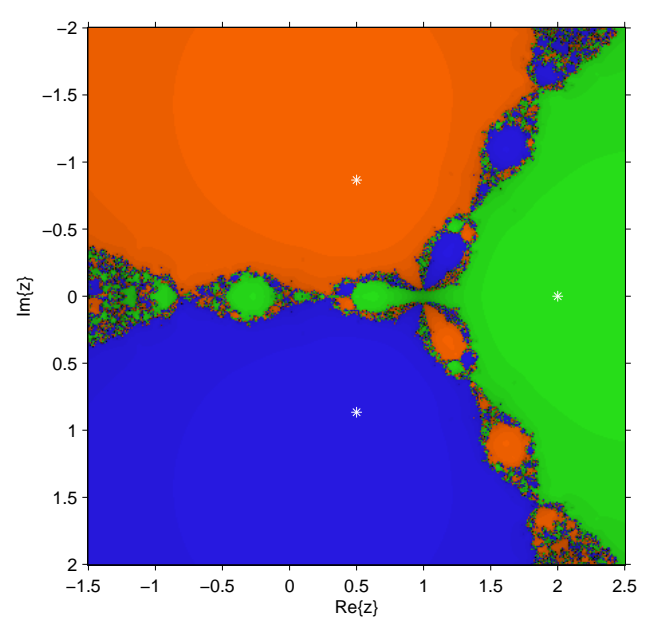

(a) M7

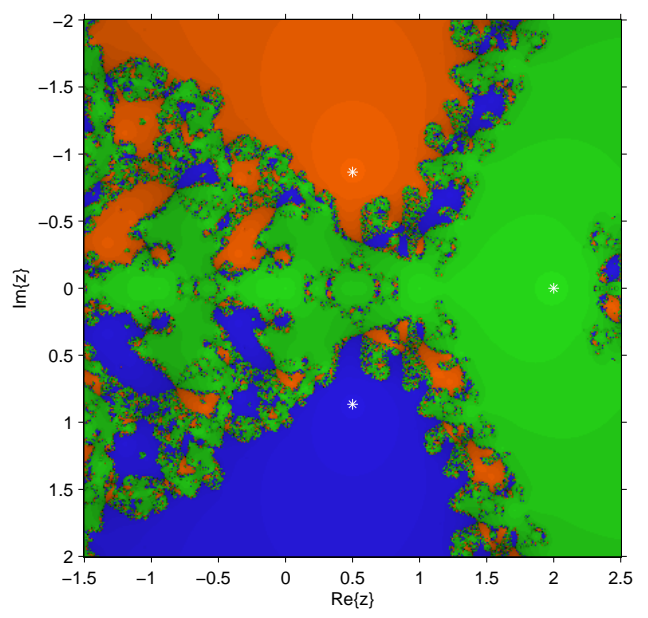

(c) $\mathrm{T} 8$

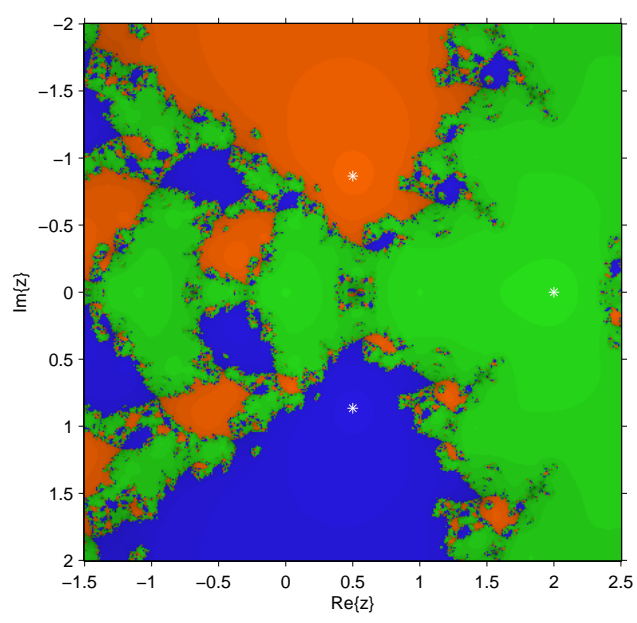

(b) ZLH8

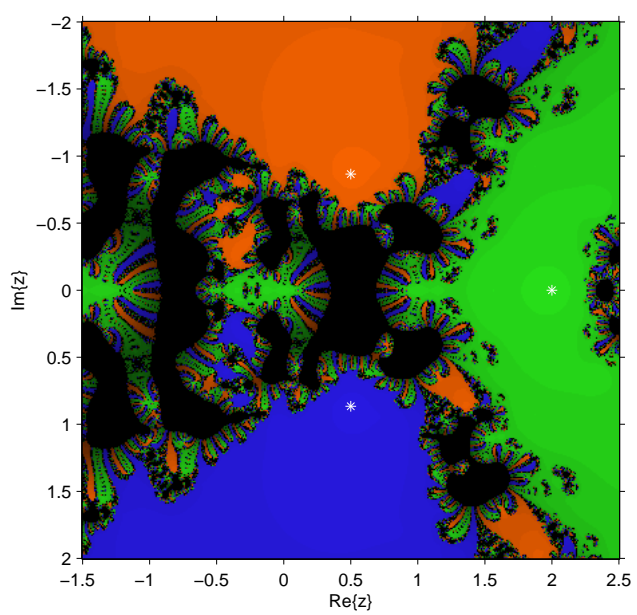

(d) S8

Figure 2: Dynamical planes of different schemes on $f_{5}(z)=(z-1)^{3}-1$

[4] J. Džunić, On efficient two-parameter methods for solving nonlinear equations, Numerical Algorithms 63(3), 549-569 (2013)

[5] J. Džunić, M.S. Petković, On generalized multipoint root-solvers with memory, Journal of Computational and Applied Mathematics 236, 2909-2920 (2012)

[6] M.S. Petković, B. Neta, L.D. Petković, J. Džunić, Multipoint methods for solving nonlinear equations, Ed. Elsevier (2013)

[7] J.R. Sharma, R. Sharma, A new family of modified Ostrowski's methods with accelerated eighth order convergence, Numerical Algorithms 54, 445-458 (2010)

[8] F. Soleymani, S. Shateyi, Two optimal eighth-order derivative-free classes of iterative methods, Abstract and Applied Analysis, Volume 2012 (2012), Article ID 318165, 14 pages, doi: 10.1155/2012/318165

[9] F. Soleymani, R. Sharma, X. Li, E. Tohidi, An optimized derivative-free form of the Potra-Pták methods, Mathematical and Computer Modelling 56, 97-104 (2012)

[10] R. Thukral, Eighth-order iterative methods without derivatives for solving nonlinear equations, ISRN Applied Mathematics, Volume 2011 (2011), Article ID 693787, 12 pages, doi: 10.5402/2011/693787 
[11] J. F. Traub, Iterative Methods for the Solution of Equations, Prentice Hall, New York, 1964

[12] X. Wang, J. Džunić, T. Zhang, On an efficient family of derivative free three-point methods for solving nonlinear equations, Applied Mathematics and Computation 219, 1749-1760 (2012)

[13] Q. Zheng, J. Li, F. Huang, An optimal Steffensen-type family for solving nonlinear equations, Applied Mathematics and Computation 217 9592-9597 (2011)

[14] J. M. Ortega, W. G. Rheinboldt, Iterative solutions of nonlinear equations in several variables, Ed. Academic Press, New York (1970)

[15] I.O. Jay, A note on $Q$-order of convergence, BIT Nunerical Mathematics 41, 422-429 (2001)

[16] P. Blanchard, Complex Analytic Dynamics on the Riemann Sphere, Bulletin of the AMS 11(1), 85-141 (1984)

[17] F. Chicharro, A. Cordero, J.R. Torregrosa, Drawing dynamical and parameters planes of iterative families and methods, Arxiv:1307.6705 [math.NA] 